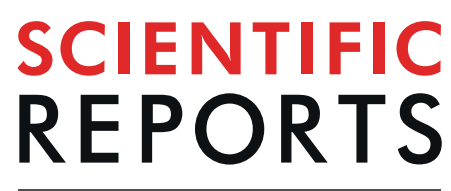

natureresearch

\title{
Genome-wide analysis of CsWOX transcription factor gene family in cucumber (Cucumis sativus L.)
}

\author{
Ran Gu ${ }^{1}$, Xiaofei Song ${ }^{2}$, Xiaofeng Liu ${ }^{1}$, Liying Yan ${ }^{3}$, Zhaoyang Zhou ${ }^{1 *}$ \& Xiaolan Zhang ${ }^{1 *}$ \\ WUSCHEL-related homeobox (WOX) transcription factors are plant-specific members that \\ characterized by the presence of a homeodomain. It has been shown that WOX members regulate \\ several aspects of plant development, but the biological functions of this CsWOX gene family remain \\ largely unknown in cucumber (Cucumis sativus L.). In this study, we identified and characterized 11 \\ putative CsWOX genes in cucumber, which are also divided into three major clades (e.g., the Ancient \\ clade, the Intermediate clade and the WUS clade). Expression pattern analysis revealed tissue-specific \\ expression patterns of CsWOX genes, including that CsWOX9 is mainly expressed in developing \\ fruit and also has lower expression in tip and axillary bud, which was further confirmed by in situ \\ hybridization assay. Moreover, overexpression of CsWOX9 in Arabidopsis led to increased branches \\ and rosette leaves, and shorter siliques. Together, these results indicated that CsWOX members may \\ regulate cucumber growth and development.
}

WUSCHEL-related homeobox (WOX) gene family are plant-specific transcription factors, which belong to homeobox superfamily. All WOX members contain a homeodomain with 60-66 amino acid residues. According to evolutionary relationships, they can be divided into WUS, Intermediate and Ancient clades ${ }^{1-3}$. These WOX members have been identified and studied in many species including Arabidopsis, rice, maize and soybean.

So far, it has been found that WOX transcription factors play important roles in regulating many aspects of growth and development including meristematic stem cell maintenance, embryonic development and polarization, lateral organ development, and organ regeneration. The founding member AtWUS is expressed in stem apical meristem (SAM) and is necessary for SAM formation and maintenance ${ }^{2,4-6}$. In addition to AtWUS, other WOX members are also involved in stem cell maintenance. For example, AtWOX5 is enriched in the quiescent center and plays an important role in maintaining the stability of stem cells of root apical meristem, similar to AtWUS in $\mathrm{SAM}^{7}$. WOX4 is mainly expressed in the procambium and cambium, and promotes differentiation and/or maintenance of the vascular procambium in Arabidopsis and tomato ${ }^{8,9}$. WOX9 promotes the growth of SAM and is required for the maintenance of WUS expression at the shoot apex, and mediates cytokinin signaling during shoot meristem establishment ${ }^{10}$.

The involvement of WOX members in plant development is not limited to stem cell maintenance. For example, AtWOX2 contributes to early pre-embryo and cotyledon boundary formation during embryo development in the egg cell and zygote ${ }^{11}$. Consistently, PaWOX2 also likely regulates embryo cell division and/or differentiation in Picea abies ${ }^{12}$. AtWOX7 is expressed at all stages of lateral root development, and inhibits lateral root development in a sugar-dependent manner ${ }^{13}$. Similarly, OsWOX6 and OsWOX11 are expressed asymmetrically in response to auxin control rice tiller angle ${ }^{14}$. Moreover, AtWOX11 and AtWOX12 play important roles in de novo root organogenesis. In responds to a wounding-induced auxin maximum, AtWOX11 and AtWOX12 promote the first-step cell fate transition from a leaf procambium to a root founder cell, initiating adventitious root organogenesis ${ }^{15}$. It has been reported that $A t W O X 13$ is mainly expressed in meristematic tissues including the replum and promotes replum development and fruit opening ${ }^{16,17}$.

\footnotetext{
${ }^{1}$ State Key Laboratories of Agrobiotechnology, Beijing Key Laboratory of Growth and Developmental Regulation for Protected Vegetable Crops, MOE Joint Laboratory for International Cooperation in Crop Molecular Breeding, China Agricultural University, Beijing, 100193, China. ${ }^{2}$ Analysis and Testing Centre, Hebei Normal University of Science \& Technology, Qinhuangdao, 066004, China. ${ }^{3}$ College of Horticulture Science and Technology, Hebei Normal University of Science\& Technology, Qinhuangdao, 066004, China. *email: zyzhou@cau.edu.cn; zhxiaolan@cau.edu.cn
} 


\begin{tabular}{|l|l|l|l|l|l|l|}
\hline Name & Gene ID & Genomic Location & Clade & AA & $\begin{array}{l}\text { MW } \\
(\mathbf{k D a})\end{array}$ & pI \\
\hline CsWUS & Csa6G505860 & $25736729 . .25738425$ & WUS Clade & 304 & 33.41 & 6.25 \\
\hline CsWOX1a & Csa1G042780 & $4494646 . .4497727$ & WUS Clade & 387 & 43.64 & 5.76 \\
\hline CsWOX1b & Csa1G025040 & $2675438 . .2678242$ & WUS Clade & 334 & 39.12 & 8.49 \\
\hline CsWOX2 & Csa1G505930 & $17913741 . .17915174$ & WUS Clade & 239 & 26.88 & 6.66 \\
\hline CsWOX3 & Csa6G301060 & $14353366 . .14354731$ & WUS Clade & 193 & 22.37 & 6.45 \\
\hline CsWOX4 & Csa2G356610 & $16722291 . .16723235$ & WUS Clade & 227 & 25.88 & 8.94 \\
\hline CsWOX7 & Csa6G010010 & $1117959 . .1118864$ & WUS Clade & 130 & 14.66 & 10.08 \\
\hline CsWOX9 & Csa6G518270 & $27456068 . .27458240$ & Intermediate Clade & 376 & 41.11 & 8.33 \\
\hline CsWOX11 & Csa3G812740 & $31266731 . .31269991$ & Intermediate Clade & 257 & 27.33 & 5.97 \\
\hline CsWOX13a & Csa3G002330 & $249427 . .252960$ & Ancient Clade & 282 & 32.11 & 6.00 \\
\hline CsWOX13b & Csa4G663700 & $23044894 . .23046632$ & Ancient Clade & 269 & 30.64 & 5.54 \\
\hline
\end{tabular}

Table 1. Characteristics of CsWOX transcription factors in cucumber.

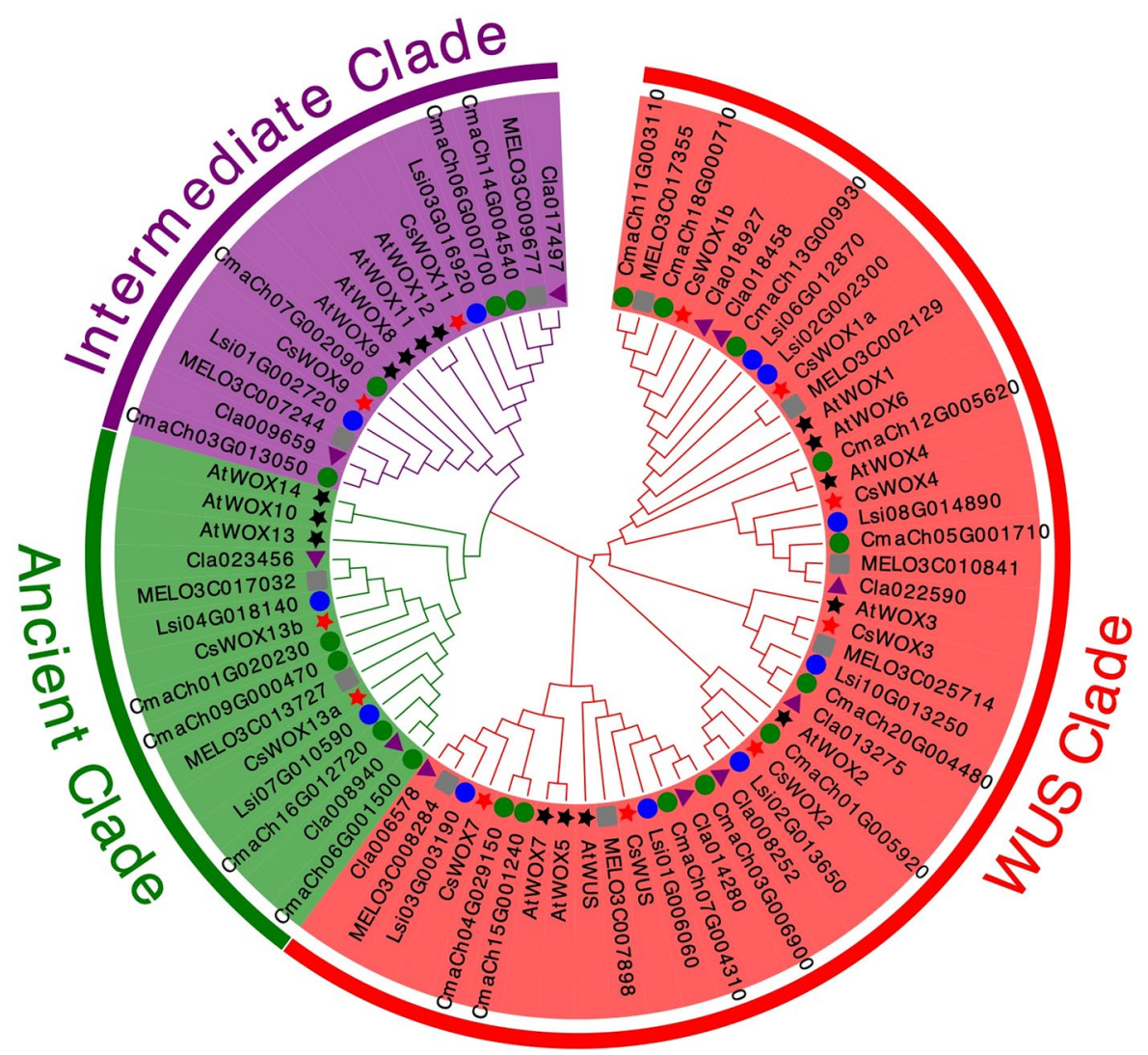

Figure 1. Phylogenetic clustering of WOX members. The full-length sequences of WOX members were used to generate the phylogenetic tree using MEGA. Arabidopsis (15 genes), cucumber (11 genes), melon (10 genes), watermelon (11 genes), pumpkin (19 genes) and calabash (11 genes) are indicated with black stars, red stars, grey rectangles, purple triangles, green circles and blue circles, respectively. Each of the three WOX clades is indicated in a specific color.

Cucumber (Cucumis sativus L.) is one of the important economic vegetable crops. Many growth and development processes affect cucumber quality and yield. Although WOX members have been shown to regulate many aspects of development in several plant species, only CsWOX1 was reported to regulate early reproductive organ development in cucumber ${ }^{18}$. It is unknown whether CsWOX members regulate different biological processes in cucumber. Here, we identified and characterized 11 putative CsWOX genes. To investigate the potential functions of CsWOX genes in cucumber, gene expression analysis showed that these CsWOX genes have different tissue-specific expression patterns. When introduced CsWOX 9 driven by the $35 S$ promoter into the Arabidopsis, the transgenic lines exhibited shorter siliques and increased branches, consistent with its expression pattern in cucumber. Together, these results indicated that CsWOX members may regulate development in cucumber. 
A

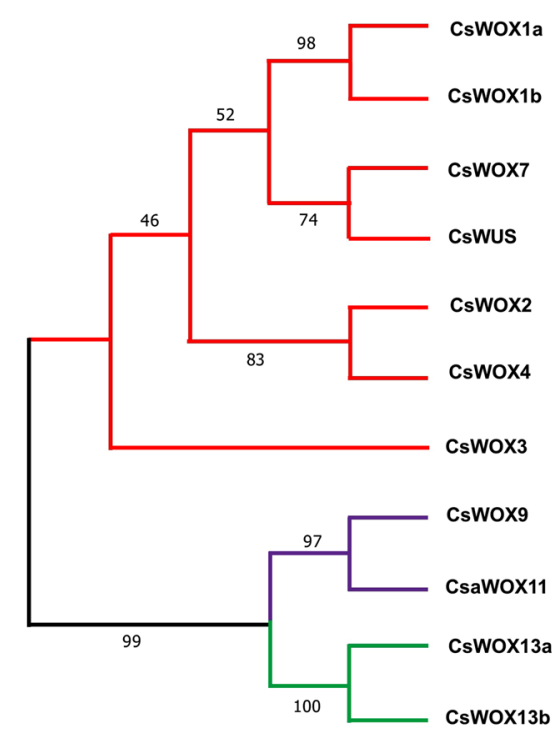

B

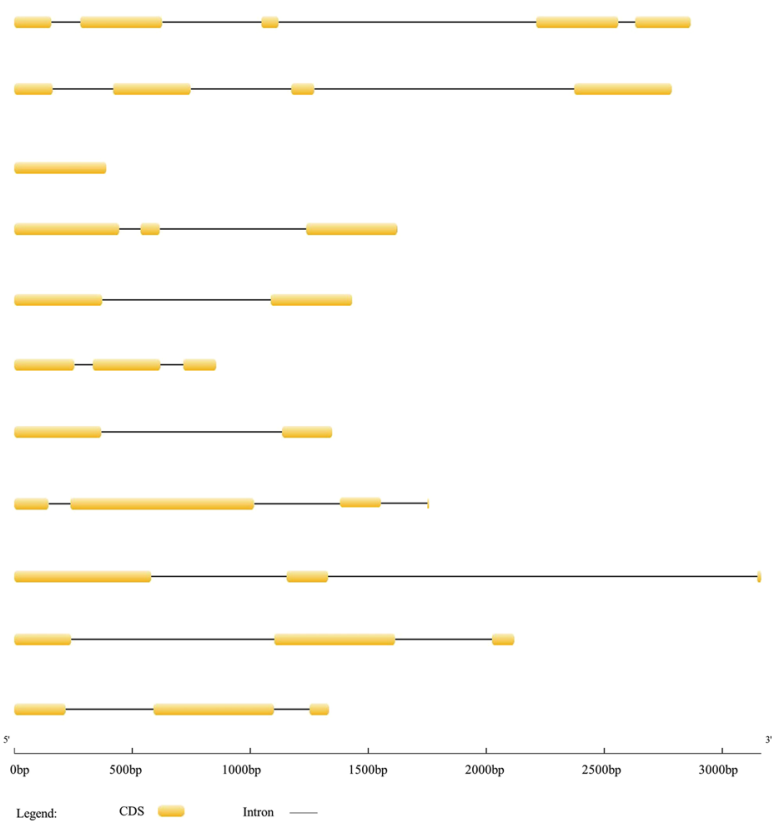

Figure 2. Phylogenetic clustering and gene structures of CsWOX members in cucumber. (A) The phylogenetic tree was generated based on the full-length sequences of cucumber CsWOX proteins. WUS Clade, Intermediate Clade and Ancient Clade are indicated in red, purple and green colors, respectively. (B) Exon-intron distribution of cucumber CsWOX genes. Yellow boxes indicate exons and black lines indicate introns.

\section{Materials and methods}

Plant materials. Cucumber (Cucumis stativus L.) inbred R461 were grown in a standard glass greenhouse of China Agricultural University (Beijing, China). Irrigation and pest control were carried out according to standard procedures. The Arabidopsis Columbia $(\mathrm{Col})$ was used as wild-type (WT) and grown at $22^{\circ} \mathrm{C}$ in a greenhouse with a 16-h-light/8-h-dark photoperiod.

Gene identification. To identify CsWOX genes in the cucumber genome (http://cucurbitgenomics.org), the full-length sequences of 15 AtWOX members obtained from Arabidopsis genome database (https://www.arabidopsis.org) were used as queries for BLASTp searches. Subsequently, the sequences of the candidate members were analyzed using SMART (http://smart.embl-heidelberg.de) and Pfam (http://pfam.xfam.org) to exclude the members that did not contain the complete homeodomain.

Bioinformatics analysis of CsWOX family members. The amino acid sequences of CsWOX members in cucumber were obtained from the Cucumber Genome Database (http://cucurbitgenomics.org/), and then the molecular weight and isoelectric points of these members were analyzed using ExPASy Proteomics Server (https://web.expasy.org/protparam/). The gene structure analysis was performed using the online program Gene Structure Display Server (GSDS: http://gsds.cbi.pku.edu.cn) ${ }^{19}$. The cis-acting elements in the promoters of CsWOX genes were analyzed using the PlantCARE (http://bioinformatics.psb.ugent.be/webtools/plantcare/ $\mathrm{html} /)^{20}$.

The full-length sequences of CsWOX family members were aligned using ClustalW in MEGA 5.0 and this alignment was used to generate the phylogenetic tree with Neighbor-Joining (NJ) method. Poisson correction, pairwise deletion and a bootstrap were conducted with 1000 replicates $^{21}$.

Gene expression analysis. Total RNA was extracted using a Quick RNA Isolation Kit (Waryoung, Beijing, China) and cDNAs were synthesized using TianScript II RT Kit (Tiangen Biotech, China). The qRT-PCR analysis was performed using ABI PRISM 7500 Real-Time PCR System (Applied Biosystems). The cucumber UBIQUITIN (Csa000874) and Arabidopsis ACTIN2 (AT3G18780) genes were used as internal controls. Three biological replicates were performed. Sequences of all the primers used in this study were shown in Table S2.

For gene expression pattern analysis, the cucumber root, stem, leaf, tip and tendril of 16-day-old seedlings, axillary bud from the top of the lateral branch, male and female buds at the $10^{\text {th }}$ stage of flower development, male and female flowers at anthesis, $1.5 \mathrm{~cm}$ fruit, $2 \mathrm{~cm}$ fruit and fruit on the day of blooming were harvested for RNA isolation.

For analysis of $A B A$ and auxin-induced gene expression, the two-week-old cucumber seedlings were treated with $100 \mu \mathrm{M}$ abscisic acid (ABA) or $100 \mu \mathrm{M}$ indole-3-acetic acid (IAA). The seedling tips were collected at $0,0.5$, $1,3,6,9,12$ and $24 \mathrm{~h}$ after treatment. 


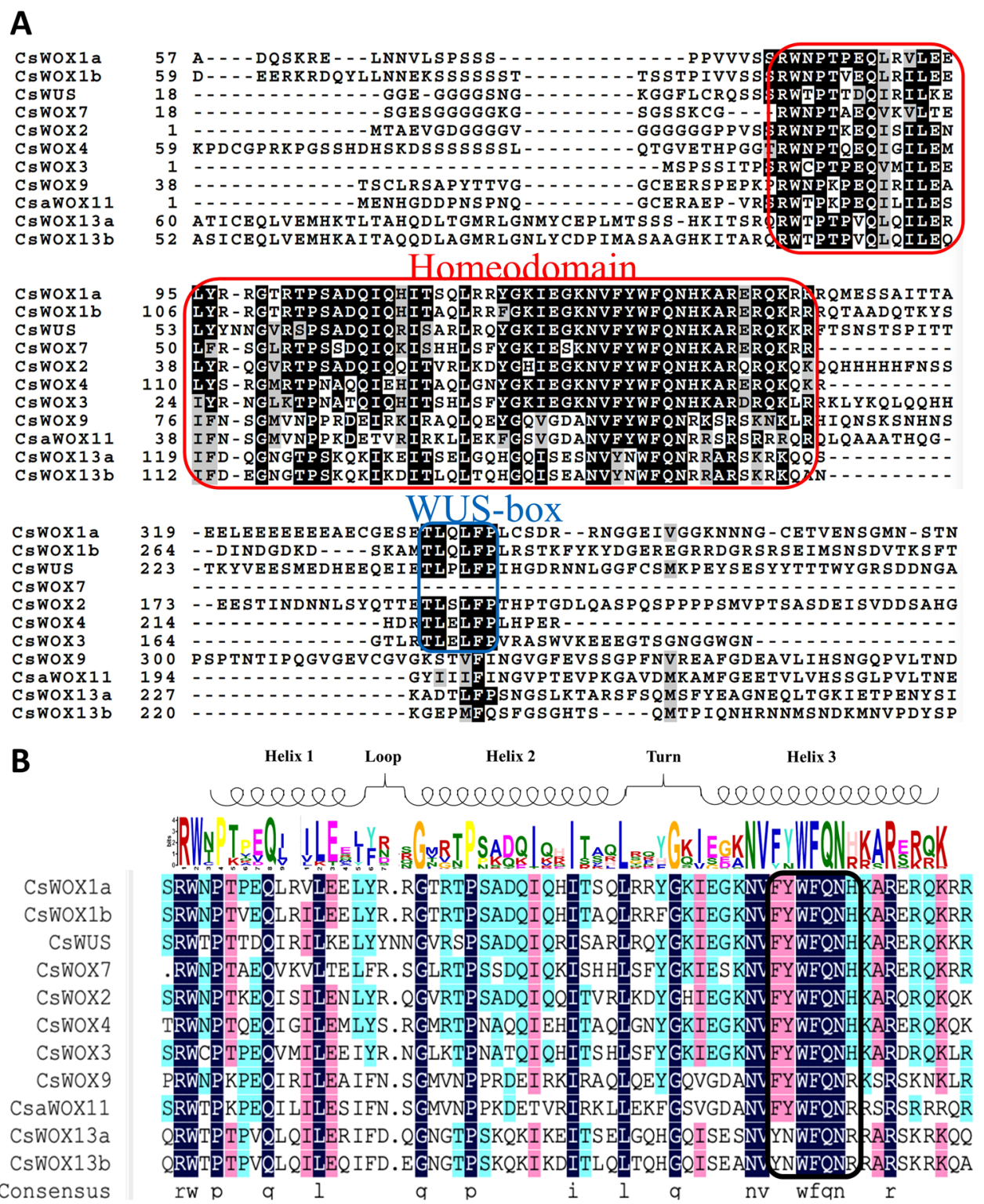

Figure 3. Protein sequence and conserved homeodomain analysis of CsWOX family members. (A) The sequence alignment of CsWOX members in cucumber. The red box indicates homeodomain and the blue box represents WUS-box domain. (B) Sequence alignment of homeodomain of CsWOX proteins in cucumber. The alignment was colored according to percent identity.

In situ hybridization. The shoot tips of 16-, 18-, and 20-day-old seedlings, young floral buds and young fruits were fixed in $3.7 \%$ FAA (3.7\% formaldehyde, $5 \%$ glacial acetic acid, and $50 \%$ ethanol $)^{22}$, and then embedded, sectioned and hybridized with digoxigenin (DIG)-labeled sense and antisense gene-specific probes as previous described ${ }^{23}$. The primer information for probe was listed in Table S2.

Arabidopsis transformation. To generate the CsWOX9 overexpression transgenic plants, the CsWOX9 coding sequence was cloned into the pBI121 vector. The resultant construct was introduced into Agrobacterium strain C58 by electroporation and transformed into Arabidopsis plants using the floral-dip method ${ }^{24}$. Primary transformants were screened on Murashige and Skoog (MS) medium with $40 \mathrm{mg} \cdot \mathrm{L}^{-1}$ kanamycin. The primers used for vector construction were listed in Table S2.

\section{Results}

Identification and phylogenetic analysis of CsWOX genes in cucumber. To identify CsWOX members in cucumber, we performed BLASTp searches using the full-length sequences of 15 Arabidopsis AtWOX members and analyzed homeodomain using SMART software ${ }^{11}$. Consistent with previous study ${ }^{18}, 11$ putative CsWOX genes were identified in cucumber (Table 1). These genes encoded proteins ranging from 130 and 387AA (amino acids) while isoelectric point (pI) values ranging from 5.54 to 10.08 , and the molecular weights from 14.66 to $43.64 \mathrm{kDa}$ (Table 1). 

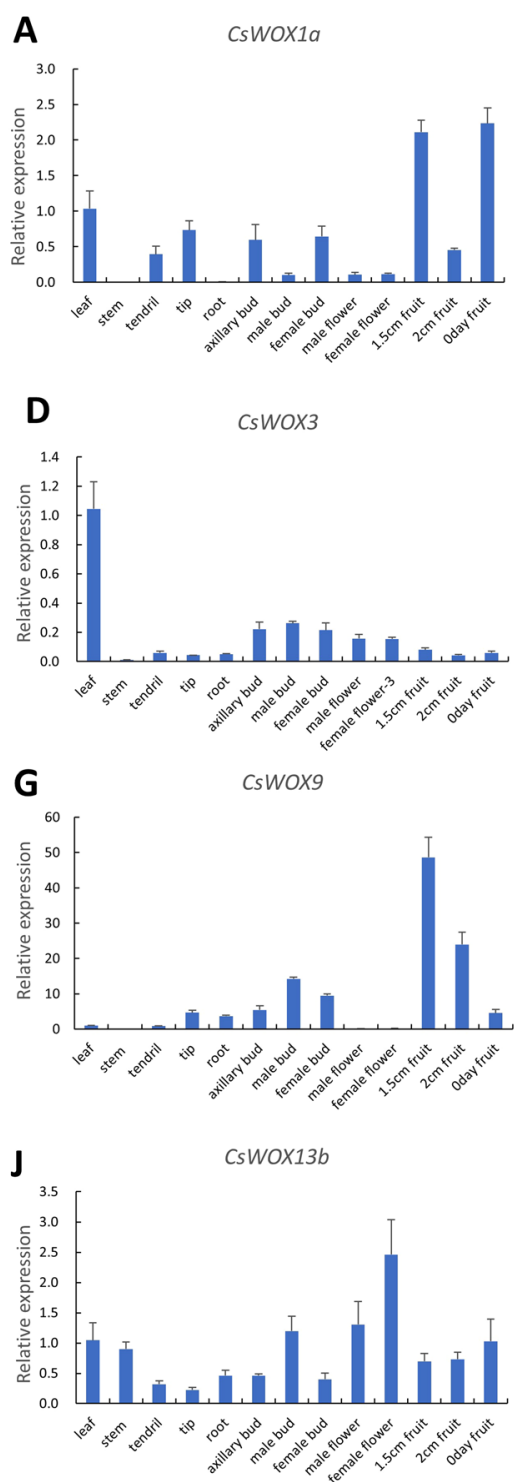

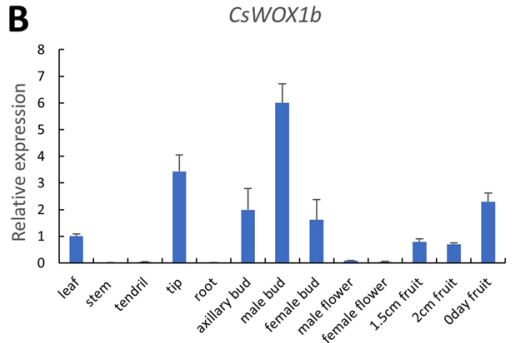

$E$

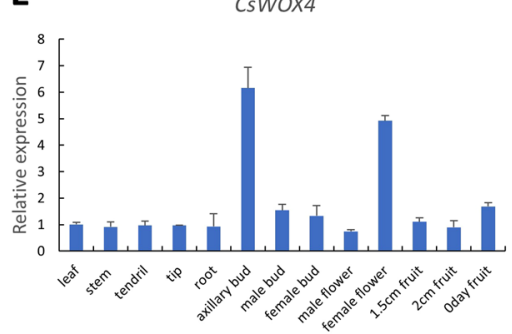

H

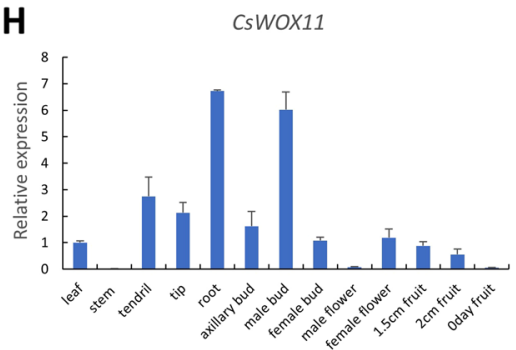

K

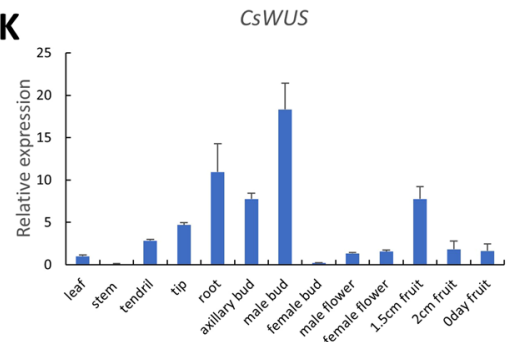

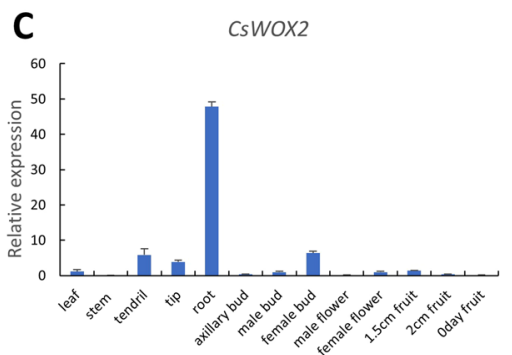

$\mathbf{F}$
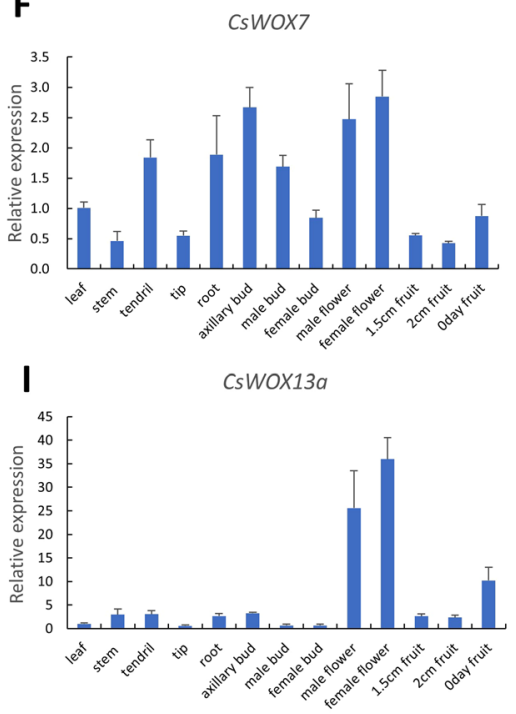

Figure 4. Expression patterns of $C s W O X$ genes in cucumber. (A-K) Tissue-specific of $C s W O X$ expression in cucumber was examined using qRT-PCR. The cucumber UBIQUITIN gene was used as an internal standard. Values are means \pm sd of three biological replicates.

To explore the evolutionary relationship of cucurbit and Arabidopsis WOX members, the full-length sequences of these members were used to generate the phylogenetic tree using MEGA5.0. These cucurbit members were renamed according to the original Arabidopsis names clustered in the same clade. The WOX members in every species can be divided into three clades, which were the Ancient clade, Intermediate clade and WUS clade. The WUS clade was the largest one in the tree and also remained the largest group in Arabidopsis and each cucurbit. While the Ancient clade only contains 15 WOX members and is the smallest clade in each tested species (Figs. 1 and 2A), indicating that WOX members between Arabidopsis and cucurbit species may have a close evolutionary relationship.

CsWOX gene structures and conserved domains. To better understand the CsWOX gene structure in cucumber, the exon/intron organization was analyzed. The results showed that all CsWOX genes contain one to five exons (Fig. 2B). Interestingly, both CsWOX13a/13b in the Ancient clade has three exons. The exon number of CsWOX genes is variable in WUS and Intermediate clades. For example, CsWOX1a contains five exons, while CsWOX7 only has a single exon (Fig. 2B).

The WOX members are plant-specific proteins containing a conserved homeodomain ${ }^{25,26}$. To study whether the domain is conserved in CsWOX members, the sequences of these proteins were aligned to generate sequence logos. The alignment results showed that all the CsWOX members contain a conserved homeodomain that carrying a helix-loop-helix-turn-helix structure (Fig. 3). Consistent with previous studies ${ }^{27,28}$, the representative motifs YNWFQNR, FYWFQNR and FYWFQNH also exist in homeodomains from the Ancient, Intermediate 
A

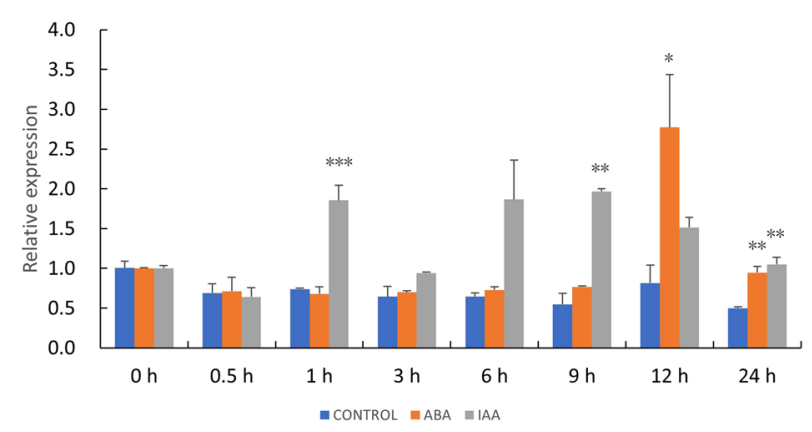

B

CsWOX3
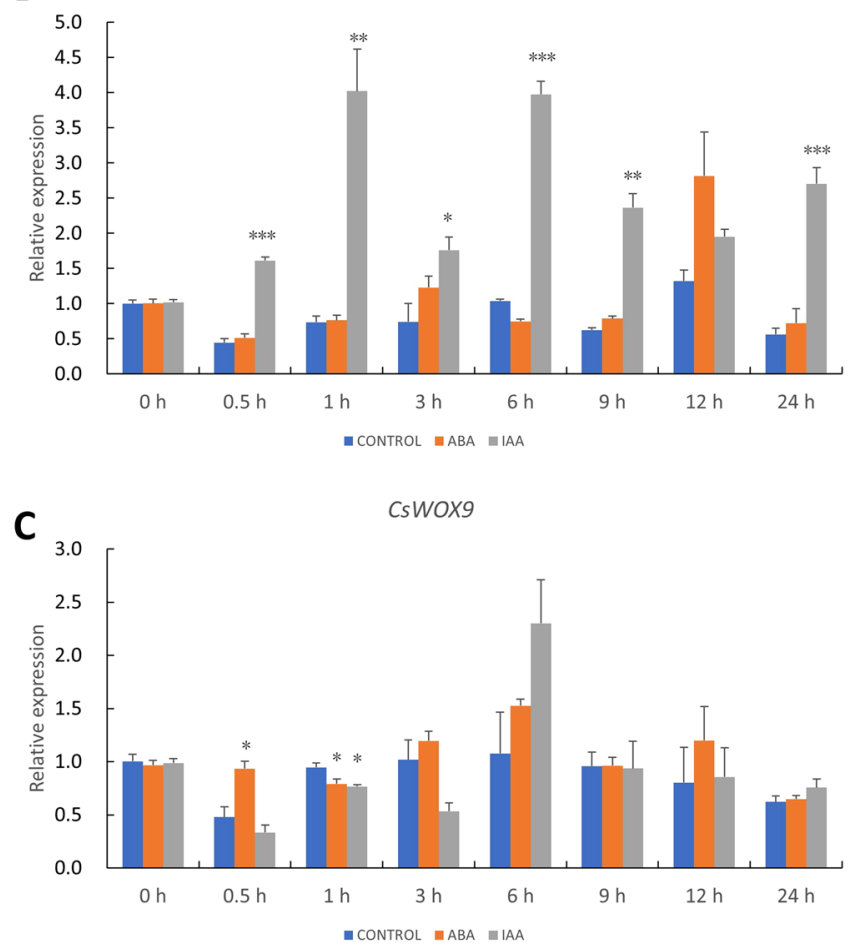

Figure 5. Hormonal regulation of $C s W O X$ expression. Two-week-old cucumber seedlings were treated with $100 \mu \mathrm{M}$ ABA or $100 \mu \mathrm{M}$ IAA. The expressions of CsWOX1b (A), CsWOX3 (B) and CsWOX9 (C) were examined at $0 \mathrm{~h}, 0.5 \mathrm{~h}, 1 \mathrm{~h}, 3 \mathrm{~h}, 6 \mathrm{~h}, 9 \mathrm{~h}, 12 \mathrm{~h}$ and $24 \mathrm{~h}$ after treatment. Values are means \pm sd of three biological replicates, and significant differences between the treated seedlings and control are indicated by asterisks $(* \mathrm{P}<0.05$ and $* * \mathrm{P}<0.01, * * * \mathrm{P}<0.001$, one-way ANOVA, Tukey post-test, three independent experiment).

and WUS clades, respectively (Fig. 3B). Moreover, the CsWOX members in WUS clade also contain a WUS-box, except CsWOX7 (Fig. 3A). While CsWOX members in Ancient and Intermediate clades have no WUS-box, indicating that these CsWOX members in different clades may be involved in different biological processes in cucumber.

Expression pattern of CsWOXs in cucumber. To investigate the details of tissue-specific expression of the $C s W O X$ genes in cucumber, the expression patterns of all 11 CsWOX genes were examined by quantitative RT-PCR (qRT-PCR) in thirteen tissues/organs, i.e., leaf, stem, tendril, tip, axillary bud, root, male bud, female bud, male flower, female flower, $1.5 \mathrm{~cm}$ fruit, $2 \mathrm{~cm}$ fruit and fruit on the day of blooming (0 day fruit). The results showed that CsWOX2 was mainly expressed in root; CsWOX3 was specifically expressed in leaf; CsWOX4 was specifically enriched in axillary bud and female flower; and CsWOX13a was highly accumulated in male and female flowers, while CsWOX1a, CsWOX1b, CsWOX7, CsWOX11, CsWOX13b and CsWUS showed no obvious organ-specific expression patterns (Fig. 4). Interestingly, CsWOX9 was mainly expressed in different developmental stages of fruit and also has lower expression in tip and axillary bud (Fig. 4G). These results suggested that CsWOX members may function in many aspects of cucumber growth and development. 

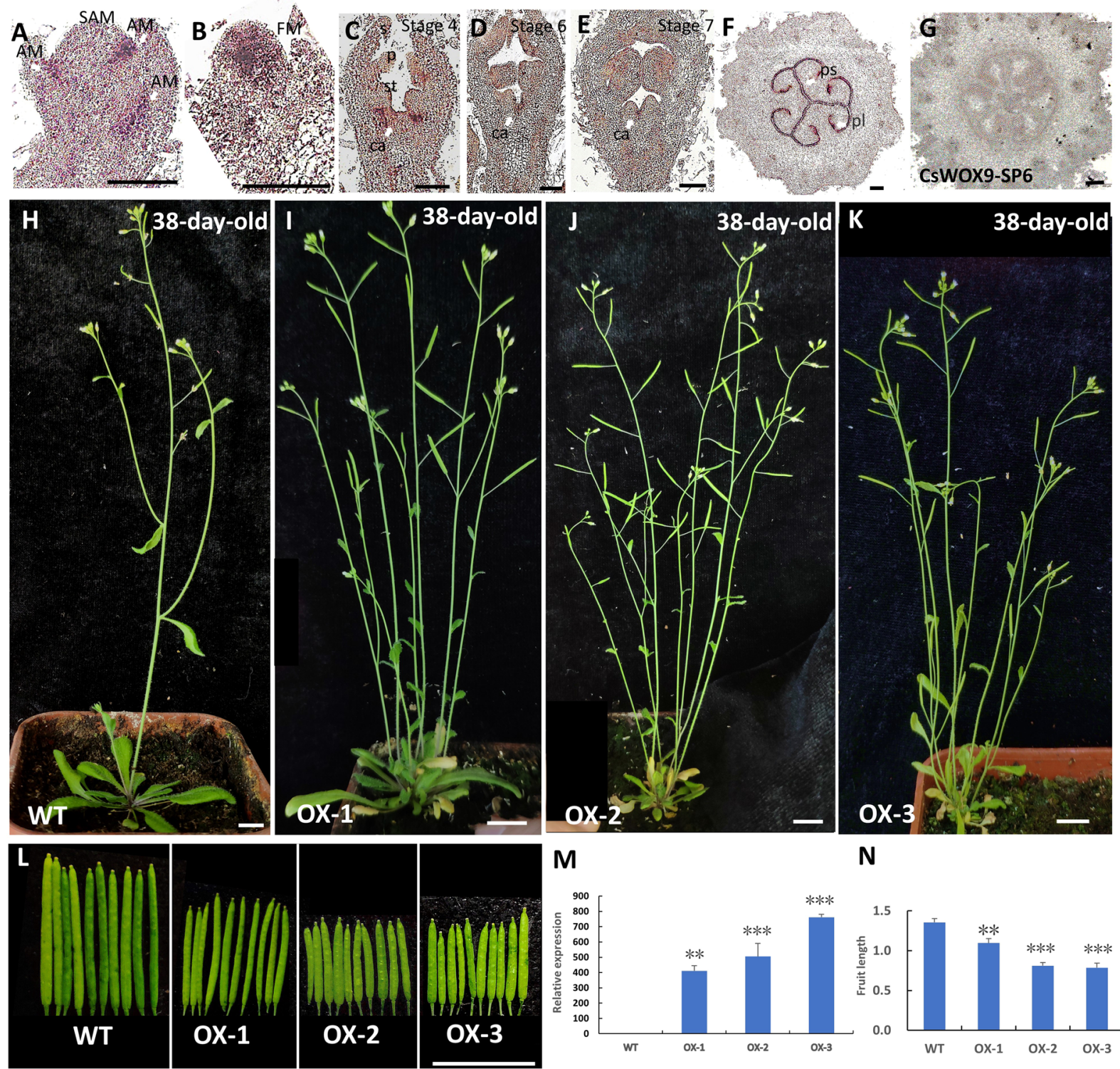

$\mathbf{N}$

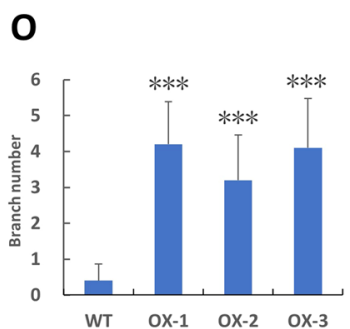

$\mathbf{P}$

Q
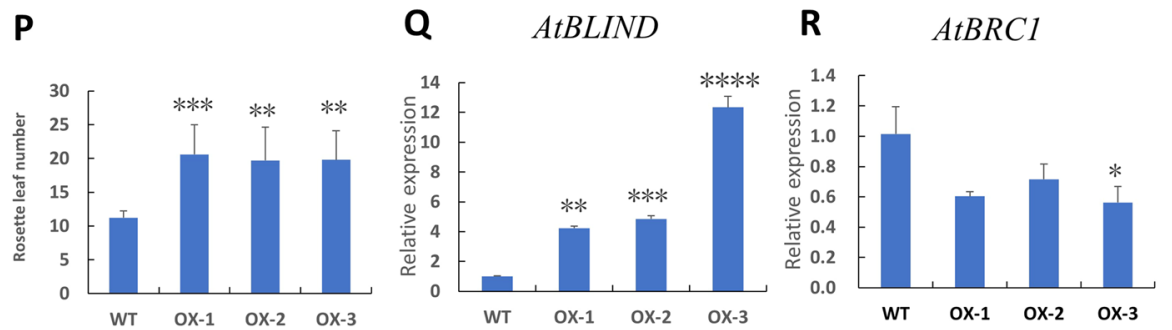

Figure 6. Ectopic expression of CsWOX9 in the wild type (WT) Arabidopsis. (A-G) In situ hybridization analysis of CsWOX9. Negative control of CsWOX9 hybridized with the sense probe in the fruit (G). SAM, Stem Apical Meristem; AM, Axillary Meristem; s, sepal; p, petal; st, stamen, ca, carpel, ps, pseudoseptum; pl, placenta. Scale bar, $100 \mu \mathrm{m}$. (H-K) Morphology of 38-day-old WT and CsWOX9 transgenic plants. (L) Siliques from the primary inflorescence of WT and CsWOX9 transgenic plants. (M) Expression analysis of CsWOX9 in WT and CsWOX9 transgenic lines. (N-P) Statistical analyses of fruit length $(\mathbf{N})$, branch number $(\mathbf{O})$ and rosette leaf number $(\mathbf{P})$ in WT and CsWOX9 transgenic plants. $(\mathbf{Q}, \mathbf{R})$ The expression of AtBLIND $(\mathbf{Q})$ and AtBRC1 $(\mathbf{R})$ in WT and CsWOX 9 transgenic plants. Values are means $\pm \mathrm{sd}$, and significant differences between the transgenic plants and WT are indicated by asterisks $(* \mathrm{P}<0.05, * * \mathrm{P}<0.01, * * * \mathrm{P}<0.001$ and $* * * * \mathrm{P}<0.0001$, one-way ANOVA, Tukey post-test, three independent experiment). 
CsWOX gene expression in response to different hormonal treatments. Plant hormones have been shown to regulate plant development. To investigate whether CsWOX genes are regulated by plant hormones, the promoter sequences ( $2000 \mathrm{bp}$ upstream of the start codon) of CsWOXs were analyzed by PlantCARE. Many cis-elements were detected in the CsWOX gene promoter regions including some respond to auxin, abscisic acid (ABA), gibberellin (GA), salicylic acid (SA) and Methyl jasmonate (MeJA) (Table S1). Previous studies showed that auxin and ABA regulated WOX expression in rice and Cunninghamia lanceolate ${ }^{29,30}$. To further confirm that the expression of CsWOX genes is also regulated by plant hormones, these members (CsWOX1b, CsWOX 3 and $C s W O X 9$ ) carrying auxin or/and ABA response elements in their promoters were selected for study. As expected, our results showed that the expression of $C s W O X 1 b$ and $C s W O X 3$ could be greatly increased by indole-3-acetic acid (IAA) treatment, and the transcript levels of $C s W O X 1 b$ and $C s W O X 9$ could respond rapidly to ABA treatment (Fig. 5). Together, these results suggested that some CsWOX members may be involved in different hormone-mediated development.

Ectopic expression of CsWOX9 affected plant architecture and fruit length in Arabidopsis. Given that CsWOX9 gene was significantly expressed in the developing fruit, it was selected for further study. in situ hybridization assay showed that $C s W O X 9$ mRNA accumulated in the axillary meristem (AM) and the flower meristem (FM) of shoot tips (Fig. 6A,B). CsWOX9 was also expressed in carpel primordium of flower bud at stage 4, and the joint of adjacent carpals in male flower bud at stage 6 and 7 (Fig. 6C-E). In young fruit, CsWOX9 signal was specifically detected in placenta (pl) and pseudoseptum (ps) (Fig. 6F). No signal was detected upon hybridization with the sense CsWOX9 probe (Fig. 6G).

To investigate the roles of $C s W O X 9$ in fruit development and other organ development, we further introduced the CsWOX9 driven by $35 S$ promoter into wild-type (WT) Arabidopsis. Ten independent transgenic lines were obtained and three representative lines (OX-1, 2, 3) were chosen for further study. All the transgenic plants exhibited shorter siliques (Fig. 6H-N). The transgenic line OX-3 showed shortest silique lengths compared to other transgenic lines, consistent with their highest expression of CsWOX9 (Fig. 6L-N). Interestingly, all the three transgenic plants also displayed increased branches and rosette leaves compared to WT, a result consistent with its expression in axillary bud (Figs. 4G; 6H-K,O,P). Previous studies showed that BRANCHED 1 (BRC1) inhibits shoot branching, while BLIND (BLIND) positively regulates axillary meristem formation ${ }^{31-34}$. To further confirm the role of CsWOX9 in regulating axillary bud outgrowth, we detected the expression levels of AtBRC1 and $A t B L I N D$ in $C s W O X 9$ transgenic plants. As expected, we found that the expression level of $A t B R C 1$ was strongly reduced and AtBLIND was highly increased in three transgenic lines (Fig. 6Q-R). Taken together, these results support the notion that CsWOX members may regulate many aspects of growth and development in cucumber.

\section{Discussion}

The WOX proteins function in key developmental processes in plant $\mathrm{s}^{35-37}$. However, only a few of members have been characterized in several plant species. In this study, the full length sequences of AtWOX members were used as queries for BLASTp searches, 11 CsWOX members were identified in cucumber. These CsWOX members can be also classified into three clades (e.g., the Ancient clade, the Intermediate clade and the WUS clade) (Fig. 1). As previous studies ${ }^{11}$, each CsWOX protein contains a homeodomain and all the members in WUS clade have a WUS-box domain except CsWOX7 (Fig. 3A). Interestingly, another previous study also identified the same CsWOX members in cucumber using the homeodomain sequences of AtWOX members for BLASTp searches ${ }^{18}$, indicating that the homeodomain is highly conserved in WOX members.

Expression pattern analysis of these CsWOX genes showed that a few of them were mainly expressed in specific organs, indicating that these genes may be involved in different development processes. For example, CsWOX1a mutation can lead to a mango fruit in cucumber ${ }^{18}$, which is consistent with that it was mainly enriched in fruit (Fig. 4A). In addition, CsWOX13a is accumulated in male and female flowers. Indeed, it has been reported that its homolog AtWOX13 regulated replum development and fruit opening in Arabidopsis ${ }^{16,17}$. But whether CsWOX13a regulates cucumber fruit development remains to be examined.

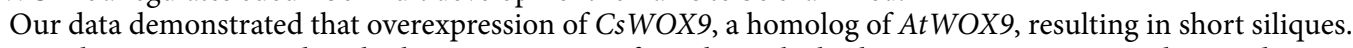
This result is consistent with its highest expression in fruit shown by both expression pattern analysis and in situ hybridization assay. But whether CsWOX9 is a regulator of fruit development in cucumber remains to be examined. Interestingly, the CsWOX9 transgenic plants also showed increased rosette leaves and branches, which is expected as its higher expression in axillary bud and axillary meristem shown by qRT-PCR and in situ hybridization. It was reported that homologs of WOX8/9 in petunia and tomato plants also contribute to inflorescence architecture by promoting the separation of lateral inflorescence meristems $\mathrm{s}^{38,39}$. Similarly, DWARF TILLER 1 (DWT1), the rice homolog of $A t W O X 8 / 9$, plays important roles in balancing branch growth ${ }^{40}$. These results suggested that the role of WOX8/9 in regulating shoot architecture is conserved in different species. Recent studies showed that auxin plays important roles in regulating fruit length and shoot branching ${ }^{32,41}$. Future studies will need to unravel whether WOX9 controls branch growth by regulating auxin synthesis or/and transport.

\section{Conclusion}

Together, our study identified and characterized 11 CsWOX members in cucumber. Tissue-specific expression of these CsWOX genes indicated that they may be involved in different developmental processes. When CsWOX9 delivered into the Arabidopsis, the transgenic lines exhibited shorter siliques and increased branches. These results uncovered that these CsWOX members play important roles in growth and development.

Received: 26 December 2019; Accepted: 21 March 2020;

Published online: 10 April 2020 


\section{References}

1. Zhang, X., Zong, J., Liu, J., Yin, J. \& Zhang, D. Genome-wide analysis of WOX gene family in rice, sorghum, maize, Arabidopsis and poplar. J. Integr. Plant. Biol. 52, 1016-1026, https://doi.org/10.1111/j.1744-7909.2010.00982.x (2010).

2. Laux, T., Mayer, K. F., Berger, J. \& Jurgens, G. The WUSCHEL gene is required for shoot and floral meristem integrity in Arabidopsis. Development 122, 87-96, https://doi.org/10.1016/S0070-2153(08)60713-9 (1996).

3. Hao, Q., Zhang, L., Yang, Y., Shan, Z. \& Zhou, X. A. Genome-Wide Analysis of the WOX Gene Family and Function Exploration of GmWOX18 in Soybean. Plants (Basel) 8, https://doi.org/10.3390/plants8070215 (2019).

4. Gross-Hardt, R., Lenhard, M. \& Laux, T. WUSCHEL signaling functions in interregional communication during Arabidopsis ovule development. Genes. Dev. 16, 1129-1138, https://doi.org/10.1101/gad.225202 (2002).

5. Deyhle, F., Sarkar, A. K., Tucker, E. J. \& Laux, T. WUSCHEL regulates cell differentiation during anther development. Dev. Biol. 302, 154-159, https://doi.org/10.1016/j.ydbio.2006.09.013 (2007).

6. Mayer, K. F. et al. Role of WUSCHEL in regulating stem cell fate in the Arabidopsis shoot meristem. Cell 95, 805-815, https://doi. org/10.1016/s0092-8674(00)81703-1 (1998).

7. Sarkar, A. K. et al. Conserved factors regulate signalling in Arabidopsis thaliana shoot and root stem cell organizers. Nature 446, 811-814, https://doi.org/10.1038/nature05703 (2007).

8. Hirakawa, Y., Kondo, Y. \& Fukuda, H. TDIF peptide signaling regulates vascular stem cell proliferation via the WOX4 homeobox gene in Arabidopsis. Plant. Cell 22, 2618-2629, https://doi.org/10.1105/tpc.110.076083 (2010).

9. Ji, J. et al. WOX4 Promotes Procambial Development. Plant. Physiol. 152, 1346-1356, https://doi.org/10.1104/pp.109.149641 (2010).

10. Skylar, A., Hong, F., Chory, J., Weigel, D. \& Wu, X. STIMPY mediates cytokinin signaling during shoot meristem establishment in Arabidopsis seedlings. Development 137, 541-549, https://doi.org/10.1242/dev.041426 (2010).

11. Haecker, A. et al. Expression dynamics of WOX genes mark cell fate decisions during early embryonic patterning in Arabidopsis thaliana. Development 131, 657-668, https://doi.org/10.1242/dev.00963 (2004).

12. Palovaara, J. \& Hakman, I. Conifer WOX-related homeodomain transcription factors, developmental consideration and expression dynamic of WOX2 during Picea abies somatic embryogenesis. Plant. Mol. Biol. 66, 533-549, https://doi.org/10.1007/s11103-0089289-5 (2008).

13. Kong, D., Hao, Y. \& Cui, H. The WUSCHEL Related Homeobox Protein WOX7 Regulates the Sugar Response of Lateral Root Development in Arabidopsis thaliana. Mol. Plant. 9, 261-270, https://doi.org/10.1016/j.molp.2015.11.006 (2016).

14. Zhang, N. et al. A Core Regulatory Pathway Controlling Rice Tiller Angle Mediated by the LAZY1-Dependent Asymmetric Distribution of Auxin. Plant. Cell 30, 1461-1475, https://doi.org/10.1105/tpc.18.00063 (2018).

15. Liu, J. et al. WOX11 and 12 are involved in the first-step cell fate transition during de novo root organogenesis in Arabidopsis. Plant. Cell 26, 1081-1093, https://doi.org/10.1105/tpc.114.122887 (2014).

16. Deveaux, Y. et al. Genes of the most conserved WOX clade in plants affect root and flower development in Arabidopsis. BMC Evol. Biol. 8, 291, https://doi.org/10.1186/1471-2148-8-291 (2008).

17. Romera-Branchat, M., Ripoll, J. J., Yanofsky, M. F. \& Pelaz, S. The WOX13 homeobox gene promotes replum formation in the Arabidopsis thaliana fruit. Plant. J. 73, 37-49, https://doi.org/10.1111/tpj.12010 (2013).

18. Niu, H. et al. The WUSCHEL-related homeobox 1 gene of cucumber regulates reproductive organ development. J. Exp. Bot. 69, 5373-5387, https://doi.org/10.1093/jxb/ery329 (2018).

19. $\mathrm{Hu}, \mathrm{B}$. et al. GSDS 2.0: an upgraded gene feature visualization server. Bioinformatics 31, 1296-1297, https://doi.org/10.1093/ bioinformatics/btu817 (2015).

20. Lescot, M. et al. PlantCARE, a database of plant cis-acting regulatory elements and a portal to tools for in silico analysis of promoter sequences. Nucleic Acids Res. 30, 325-327, https://doi.org/10.1093/nar/30.1.325 (2002).

21. Tamura, K. et al. MEGA5: molecular evolutionary genetics analysis using maximum likelihood, evolutionary distance, and maximum parsimony methods. Mol. Biol. Evol. 28, 2731-2739, https://doi.org/10.1093/molbev/msr121 (2011).

22. Bai, S. L. et al. Developmental analyses reveal early arrests of the spore-bearing parts of reproductive organs in unisexual flowers of cucumber (Cucumis sativus L.). Planta 220, 230-240, https://doi.org/10.1007/s00425-004-1342-2 (2004).

23. Zhang, X. et al. Transcription repressor HANABA TARANU controls flower development by integrating the actions of multiple hormones, floral organ specification genes, and GATA3 family genes in Arabidopsis. Plant. Cell 25, 83-101, https://doi.org/10.1105/ tpc.112.107854 (2013).

24. Clough, S. J. \& Bent, A. F. Floral dip: a simplified method for Agrobacterium-mediated transformation of Arabidopsis thaliana. Plant. J. 16, 735-743, https://doi.org/10.1046/j.1365-313x.1998.00343.x (1998).

25. van der Graaff, E., Laux, T. \& Rensing, S. A. The WUS homeobox-containing (WOX) protein family. Genome Biol. 10, 248, https:// doi.org/10.1186/gb-2009-10-12-248 (2009).

26. Gehring, W. J., Affolter, M. \& Bürglin, T. Homeodomain Proteins. Annu. Rev. Biochem. 63, 487-526, https://doi.org/10.1146/ annurev.bi.63.070194.002415 (1994).

27. Nardmann, J. \& Werr, W. The invention of WUS-like stem cell-promoting functions in plants predates leptosporangiate ferns. Plant. Mol. Biol. 78, 123-134, https://doi.org/10.1007/s11103-011-9851-4 (2012).

28. Ikeda, M., Mitsuda, N. \& Ohme-Takagi, M. Arabidopsis WUSCHEL is a bifunctional transcription factor that acts as a repressor in stem cell regulation and as an activator in floral patterning. Plant. Cell 21, 3493-3505, https://doi.org/10.1105/tpc.109.069997 (2009).

29. Zhou, X. et al. Desiccation Treatment and Endogenous IAA Levels Are Key Factors Influencing High Frequency Somatic Embryogenesis in Cunninghamia lanceolata (Lamb.) Hook. Front. Plant. Sci. 8, 2054, https://doi.org/10.3389/fpls.2017.02054 (2017).

30. Cheng, S., Huang, Y., Zhu, N. \& Zhao, Y. The rice WUSCHEL-related homeobox genes are involved in reproductive organ development, hormone signaling and abiotic stress response. Gene 549, 266-274, https://doi.org/10.1016/j.gene.2014.08.003 (2014).

31. Minakuchi, K. et al. FINE CULM1 (FC1) works downstream of strigolactones to inhibit the outgrowth of axillary buds in rice. Plant. Cell Physiol. 51, 1127-1135, https://doi.org/10.1093/pcp/pcq083 (2010).

32. Shen, J. et al. CsBRC1 inhibits axillary bud outgrowth by directly repressing the auxin efflux carrier CsPIN3 in cucumber. Proc. Natl Acad. Sci. USA 116, 17105-17114, https://doi.org/10.1073/pnas.1907968116 (2019).

33. Braun, N. et al. The pea TCP transcription factor PsBRC1 acts downstream of Strigolactones to control shoot branching. Plant. Physiol. 158, 225-238, https://doi.org/10.1104/pp.111.182725 (2012).

34. Guo, D. et al. The WRKY Transcription Factor WRKY71/EXB1 Controls Shoot Branching by Transcriptionally Regulating RAX Genes in Arabidopsis. Plant. Cell 27, 3112-3127, https://doi.org/10.1105/tpc.15.00829 (2015).

35. Breuninger, H., Rikirsch, E., Hermann, M., Ueda, M. \& Laux, T. Differential expression of WOX genes mediates apical-basal axis formation in the Arabidopsis embryo. Dev. Cell 14, 867-876, https://doi.org/10.1016/j.devcel.2008.03.008 (2008).

36. Costanzo, E., Trehin, C. \& Vandenbussche, M. The role of WOX genes in flower development. Ann. Bot. 114, 1545-1553, https://doi. org/10.1093/aob/mcu123 (2014).

37. Dolzblasz, A. et al. Stem Cell Regulation by Arabidopsis WOX Genes. Mol. Plant. 9, 1028-1039, https://doi.org/10.1016/j. molp.2016.04.007 (2016).

38. Lippman, Z. B. et al. The making of a compound inflorescence in tomato and related nightshades. PLoS Biol. 6, e288, https://doi. org/10.1371/journal.pbio.0060288 (2008). 
39. Rebocho, A. B. et al. Role of EVERGREEN in the development of the cymose petunia inflorescence. Dev. Cell 15, 437-447, https:// doi.org/10.1016/j.devcel.2008.08.007 (2008).

40. Wang, W. et al. Dwarf Tiller1, a Wuschel-related homeobox transcription factor, is required for tiller growth in rice. PLoS Genet. 10, e1004154, https://doi.org/10.1371/journal.pgen.1004154 (2014).

41. Zhao, J. et al. A Functional Allele of CsFUL1 Regulates Fruit Length through Repressing CsSUP and Inhibiting Auxin Transport in Cucumber. Plant. Cell 31, 1289-1307, https://doi.org/10.1105/tpc.18.00905 (2019).

\section{Acknowledgements}

This study was supported by the National Natural Science Foundation of China [31772315] and [31572132], and the Construction of Beijing Science and Technology Innovation and Service Capacity in Top Subjects [CEFFPXM2019_014207_000032].

\section{Author contributions}

R.G. and X.Z. conceived and designed the experiments. R.G., X.S. and X.L. performed the experiments. R.G., L.Y., and Z.Z. analyzed the data. R.G., Z.Z., and X.Z. wrote the paper. All authors read and approved the final manuscript.

\section{Competing interests}

The authors declare no competing interests.

\section{Additional information}

Supplementary information is available for this paper at https://doi.org/10.1038/s41598-020-63197-z.

Correspondence and requests for materials should be addressed to Z.Z. or X.Z.

Reprints and permissions information is available at www.nature.com/reprints.

Publisher's note Springer Nature remains neutral with regard to jurisdictional claims in published maps and institutional affiliations.

Open Access This article is licensed under a Creative Commons Attribution 4.0 International License, which permits use, sharing, adaptation, distribution and reproduction in any medium or format, as long as you give appropriate credit to the original author(s) and the source, provide a link to the Creative Commons license, and indicate if changes were made. The images or other third party material in this article are included in the article's Creative Commons license, unless indicated otherwise in a credit line to the material. If material is not included in the article's Creative Commons license and your intended use is not permitted by statutory regulation or exceeds the permitted use, you will need to obtain permission directly from the copyright holder. To view a copy of this license, visit http://creativecommons.org/licenses/by/4.0/.

(c) The Author(s) 2020 